\title{
L'attendrissement dans la compassion et la pitié
}

Emotion in compassion and pity

Eliane Soares de Lima

\section{(2) OpenEdition}

Journals

\section{Electronic version}

URL: http://journals.openedition.org/aad/3889

DOI: 10.4000/aad.3889

ISSN: 1565-8961

\section{Publisher}

Université de Tel-Aviv

\section{Electronic reference}

Eliane Soares de Lima, "L'attendrissement dans la compassion et la pitié », Argumentation et Analyse du Discours [Online], 24 | 2020, Online since 16 April 2020, connection on 18 April 2020. URL : http:// journals.openedition.org/aad/3889; DOI : https://doi.org/10.4000/aad.3889

This text was automatically generated on 18 April 2020.

\section{(c) (i) (9)}

Argumentation \& analyse du discours est mis à disposition selon les termes de la licence Creative Commons Attribution - Pas d'Utilisation Commerciale - Pas de Modification 4.0 International. 


\title{
L'attendrissement dans la compassion et la pitié
}

Emotion in compassion and pity

\author{
Eliane Soares de Lima
}

Une des principales difficultés de l'interaction passionnelle tient justement dans le fait que, en dehors d'un parcours complet qui comprend entre autres l'expression somatique ou verbale, la passion de l'un est illisible pour l'autre : le nom de la passion fournit alors une indication de suppléance, qui réactive un schéma canonique oublié.

Jacques Fontanille (1999 : 225)

\section{L'analyse lexicale}

1 Les premiers développements de la sémiotique des passions ${ }^{1}$, sur lesquels s'appuie notre discussion, ont privilégié l'analyse lexicale pour identifier, dans les discours, les dispositions incitant le sujet à un sentiment déterminé. Ils s'attachaient au rôle de ses attentes et à celui des idéologies dans l'émergence de ses états d'âme, ainsi que dans sa manière d'être et de réagir aux choses. Ses présupposés théoriques ont porté sur l'examen des passions-lexèmes (colère, avarice, jalousie, etc.) ainsi que sur leurs expressions discursives, en suivant une logique syntagmatique susceptible de mettre à nu le processus narratif sous-jacent aux définitions.

2 Selon Greimas, les lexèmes se présentent comme des condensations de structures discursives et narratives, qui contiennent les premières informations sur les modalités de «fonctionnement» des passions. Ainsi, il explique que «les descriptions lexématiques peuvent constituer, de façon économique, des modèles de prévisibilité pour des analyses discursives ultérieures » (1983:225).

Comme le fait remarquer Bertrand (2000 : 235), il est tout à fait vrai que «les taxinomies qui modèlent les configurations passionnelles relèvent de l'usage [...]; soumises à des régimes de sensibilisation et de moralisation variables, [les passions] forment ainsi des taxinomies connotatives qui permettent d'identifier et de distinguer 
des formes culturelles» (ibid.). Par conséquent, tout en reconnaissant le relativisme culturel lié au lexique, puisque chaque langue utilise des formes variées et spécifiques pour conceptualiser des comportements pathémiques de même nature, nous avons délibérément choisi de débuter par un examen lexical notre recherche autour de l'attendrissement propre aux effets passionnels de la compassion et de la pitié. Nous estimons que ces deux passions, en dépit de leurs relations sémantiques de voisinage et d'imbrication, possèdent des particularités structurantes spécifiques, susceptibles d'être conçues comme des états d'âme distincts.

Il est bon de préciser néanmoins, eu égard à nos premières considérations, que nous traiterons la compassion et la pitié selon une perspective occidentale, imprégnée de l'héritage culturel judéo-chrétien et axiologiquement différente des points de vue enracinés dans d'autres cultures, comme le bouddhisme. Si, dans le premier cas, de tels sentiments reposent sur une identification suivie d'une certaine forme de partage, dans le second cas, le fait de considérer l'autre comme un prochain ne saurait dépendre d'une reconnaissance. Bien au contraire, le sentiment d'attendrissement qui est prêché par la doctrine bouddhiste (et qui est nommé compassion) se fonde précisément sur une annulation du « moi » au profit de l'autre, sur un détachement de soi.

Cela dit, pour nous, la signification dictionnairique de la compassion et de la pitié constitue un point d'appui pour identifier, évaluer et comprendre les caractéristiques proprement formelles des phénomènes signifiants qu'elles nomment. Nous passons ainsi en revue les définitions de ces passions-lexèmes, telles qu'elles figurent dans certains dictionnaires des principales langues romanes: le français, l'espagnol, le portugais et l'italien. Il ne s'agit cependant pas de mener une étude purement sémantique, mais plutôt d'identifier, à travers les données collectées pour les sèmes, les dispositifs ainsi que les opérations générales qui se dégagent de la production des deux noyaux pathémiques en question.

6 Nous voudrions saisir, à travers cette analyse, la (re)formulation structurante du contenu sémantique de la compassion et de la pitié, en identifiant sur cette base, comme le faisait Greimas, les organisations modales sous-jacentes et les opérations plus abstraites qui les prédisposent à participer à des configurations passionnelles plus ou moins sensibles ou intelligibles.

\section{Compassion et pitié : relevé des définitions}

7 La compassion comme la pitié, indépendamment des interprétations axiologiques qu'elles reçoivent des différentes cultures, renvoient de manière générale à l'attendrissement causé par l'observation de la souffrance manifeste d'un être vivant. Toutefois, comme le souligne Ricot (2013:9) : «la concurrence lexicale est rude pour exprimer le sentiment éprouvé devant le malheur d'autrui ». Entre la compassion et la pitié figurent le fait de plaindre quelqu'un, la commisération, la sympathie, la charité, l'apitoiement, l'attendrissement, la miséricorde, etc., « comme si cette multiplication terminologique était l'indice d'une recherche désespérée, grâce à un mot enfin approprié, d'une idée adéquate, difficile à établir en raison des écueils rencontrés autour de la notion elle-même et de son rôle dans la vie morale » (ibid.).

8 Dans la plupart des dictionnaires de langues romanes, les acceptions relevées pour ces lexèmes n'explicitent guère la nuance entre leurs signifiés et les placent au contraire comme des synonymes les uns des autres. Par exemple, les définitions de la compassion 
et de la pitié dans les dictionnaires de langue portugaise consultés, tout en admettant parfois des degrés, croisent leurs contenus et présentent un terme comme le synonyme de l'autre (voir Tableau 1).

\begin{tabular}{|c|c|}
\hline COMPAIXÃO (Compassion) & PIEDADE (Pitié) \\
\hline \multicolumn{2}{|l|}{ Acceptions dictionnairiques } \\
\hline $\begin{array}{l}n \text {. f. Sentimento que nos desperta a desgraça; pesar } \\
\text { que nos desperta o mal alheio, dó; comiseração: o } \\
\text { estado daqueles infelizes causa compaixão; [...] } \\
\text { n. f. Sentiment éprouvé devant le malheur ; } \\
\text { attendrissement suscité par le mal d'autrui, plaindre } \\
\text { (qqn.); commisération: compassion provoquée par } \\
\text { l'état des malheureux }{ }^{2} \text {. }\end{array}$ & $\begin{array}{l}n . f \text {. Devoção, amor e repeito pelas coisas } \\
\text { religiosas; religião, devoção. // Respeito } \\
\text { pelos mortos. // Sentimento inspirado pelos } \\
\text { males alheios e que se dispõe a remediá-los } \\
\text { ou a mitigá-los. // Compaixão; dó; } \\
\text { comiseração. [...] } \\
\text { n. f. Dévotion, amour et respect pour les } \\
\text { choses religieuses; religion, dévotion. // } \\
\text { Respect pour les morts. // Sentiment } \\
\text { inspiré par les maux d'autrui et qui pousse à } \\
\text { y remédier ou à les atténuer. // Compassion } \\
\text {; plaindre (qqn.); commisération. [...] }\end{array}$ \\
\hline \multicolumn{2}{|c|}{$\begin{array}{l}\text { Source : Dicionário contemporâneo da Língua Portuguesa, v. 1. 1958. Caldas Aulete, } 4 \text { éd. (Rio de Janeirc } \\
\text { : Delta) }\end{array}$} \\
\hline $\begin{array}{l}\text { s.f. sentimento piedoso de simpatia para com a } \\
\text { tragédia pessoal de outrem, acompanhado do desejo } \\
\text { de minorá-la; participação espiritual na infelicidade } \\
\text { alheia que suscita um impulso altruísta de ternura } \\
\text { para com o sofredor. } \\
\text { n. f. Sentiment d'apitoiement, de sympathie, éprouvé } \\
\text { devant le malheur d'autrui, accompagné du désir de } \\
\text { l'amoindrir ; participation spirituelle au malheur } \\
\text { d'autrui qui suscite une impulsion altruiste de } \\
\text { tendresse pour la personne qui souffre. }\end{array}$ & $\begin{array}{l}\text { s.f. } 1 \text { devoção, amor pelas coisas religiosas; } \\
\text { religiosidade; } 1.1 \text { Rubrica: religião; virtude } \\
\text { que permite render a Deus o culto que lhe é } \\
\text { devido; } 2 \text { compaixão pelo sofrimento alheio; } \\
\text { comiseração, dó, misericórdia. } \\
\text { n. f. } 1 \text { Dévotion, amour pour les choses } \\
\text { religieuses; religiosité; } 1.1 \text { Rubrique : } \\
\text { religion; vertu qui permet de rendre à Dieu } \\
\text { le culte qui lui est dû; } 2 \text { Compassion pour la } \\
\text { souffrance d'autrui ; commisération, } \\
\text { plaindre (qqn.), miséricorde. }\end{array}$ \\
\hline & \\
\hline
\end{tabular}

Tableau 1 : Tableau comparatif des définitions relevées dans les dictionnaires de portugais

9 En dépit d'un renvoi à la religiosité, à un certain "devoir religieux », dans le cas de la pitié, ce qui n'apparaît pas dans la définition de la compassion, ces deux passions "nucléaires", si l'on peut dire, sont ici clairement conçues comme étant des synonymes. La définition de la compassion présente les termes «sentiment d'apitoiement", "pitié ", tandis que celle de la pitié fait toujours référence à la compassion. Il en va de même pour les termes "périphériques" (commisération, apitoiement, miséricorde, etc.). Cependant, si nous pensons à la praxis énonciative impliquée dans l'usage de ces passions-lexèmes, leur relation de synonymie semble se diluer quelque peu. Un terme prend souvent le pas sur les autres, en fonction de la situation et de la connotation que l'on entend affirmer ou nier. 
10 Dans les dictionnaires de français (voir tableau 2), les définitions de la compassion et de la pitié indiquent plus clairement la différence de configuration de l'identification entre les sujets pour chacun des cas. Selon Ricot (2013 : 10), "la langue hésite aujourd'hui à employer le mot de pitié parce que le terme a pris, assez souvent dans l'usage, une signification de condescendance, voire de mépris et de dédain, qui semble avoir moins contaminé celui de compassion ». Tappolet $(2000: 177)$ observe également que «les exemples en français ne manquant d'ailleurs pas - il faut sans doute distinguer la pitié et la compassion ».

\begin{tabular}{|l|l|}
\hline COMPASSION & PITIÉ \\
\hline \multicolumn{2}{|l|}{ Acceptions relevées dans des dictionnaires de français } \\
\hline $\begin{array}{l}n . f .1 \text { Sentiment qui nous fait compatir. } \\
2 \text { État de celui qui est à plaindre. }\end{array}$ & $\begin{array}{l}\text { n.f. 1 Sentiment qui saisit à la vue des souffrances et } \\
\text { qui porte à les soulager. 2 Pitié, se dit quelquefois } \\
\text { en un sens où il entre quelque mépris. }\end{array}$ \\
\hline $\begin{array}{l}\text { Source : Dictionnaire Littré (en ligne). Disponible sur : http://littre.reverso.net/dictionnaire- } \\
\text { francais/definition/compassion }\end{array}$ & $\begin{array}{l}1 \text { Sympathie qui naît de la connaissance des } \\
\text { souffrances d'autrui et fait souhaiter qu'elles soient } \\
\text { soulagées } \rightarrow \text { attendrissement, commisération, } \\
\text { compassion, miséricorde. } \\
2 \text { Sentiment de commisération accompagné } \\
\text { d'appréciation défavorable ou de mépris. } \\
\text { partager les maux d'autrui } \rightarrow \text { apitoiement } \\
\text { commisération, miséricorde ; pitié. [...] } \\
\text { humanité, sensibilité. }\end{array}$ \\
\hline \begin{tabular}{l} 
Source : Dictionnaire Le Petit Robert de la langue française. CD-Rom \\
\hline
\end{tabular}
\end{tabular}

Tableau 2 : Tableau comparatif des définitions relevées dans des dictionnaires de français

11 Ces deux passions, pourtant conçues comme des états d'âme synonymes, se distinguent donc : l'acception de la compassion manifeste le sème de partage, de sensibilité, contrairement à celle de la pitié, qui mentionne notamment un certain mépris. En effet, même si les définitions dictionnairiques ne soulignent pas la différence entre ces deux passions, le sentiment d'attendrissement éprouvé devant la souffrance d'autrui peut se présenter tantôt comme naturel, irréfléchi, lorsqu'il existe un mouvement de partage de la douleur de celui qui souffre, un «sentir avec »; tantôt comme le résultat d'une évaluation moins spontanée, plus cognitive, à partir de laquelle l'observateur, qui assume une perspective extérieure et objective, compare sa situation positive à celle dysphorique du sujet souffrant, en se sensibilisant à partir d'un "sentir pour». Ce "sentir pour " ne met pas en scène la communion des sentiments, mais plutôt une relation d'altérité entre celui qui observe la souffrance et celui qui la vit. Rousseau (1969 : 319) note que « la pitié est douce parce que, en se mettant à la place de celui qui souffre, on sent pourtant le plaisir de ne pas souffrir comme lui ».

Dans les dictionnaires d'italien, quoique la compassion et la pitié apparaissent comme des synonymes, les deux types d'attendrissement y étant mentionnés, le fait contraire se produit. La pitié est en effet présentée comme l'affect euphorisé, considéré comme plus fort et pathémique, et la compassion, comme l'affect dysphorisé, associé dans la 
plupart des cas à une attitude de mépris, malgré la présence du sème " partage », du " sentir avec », comme le montre le tableau comparatif ci-dessous (voir Tableau 3):

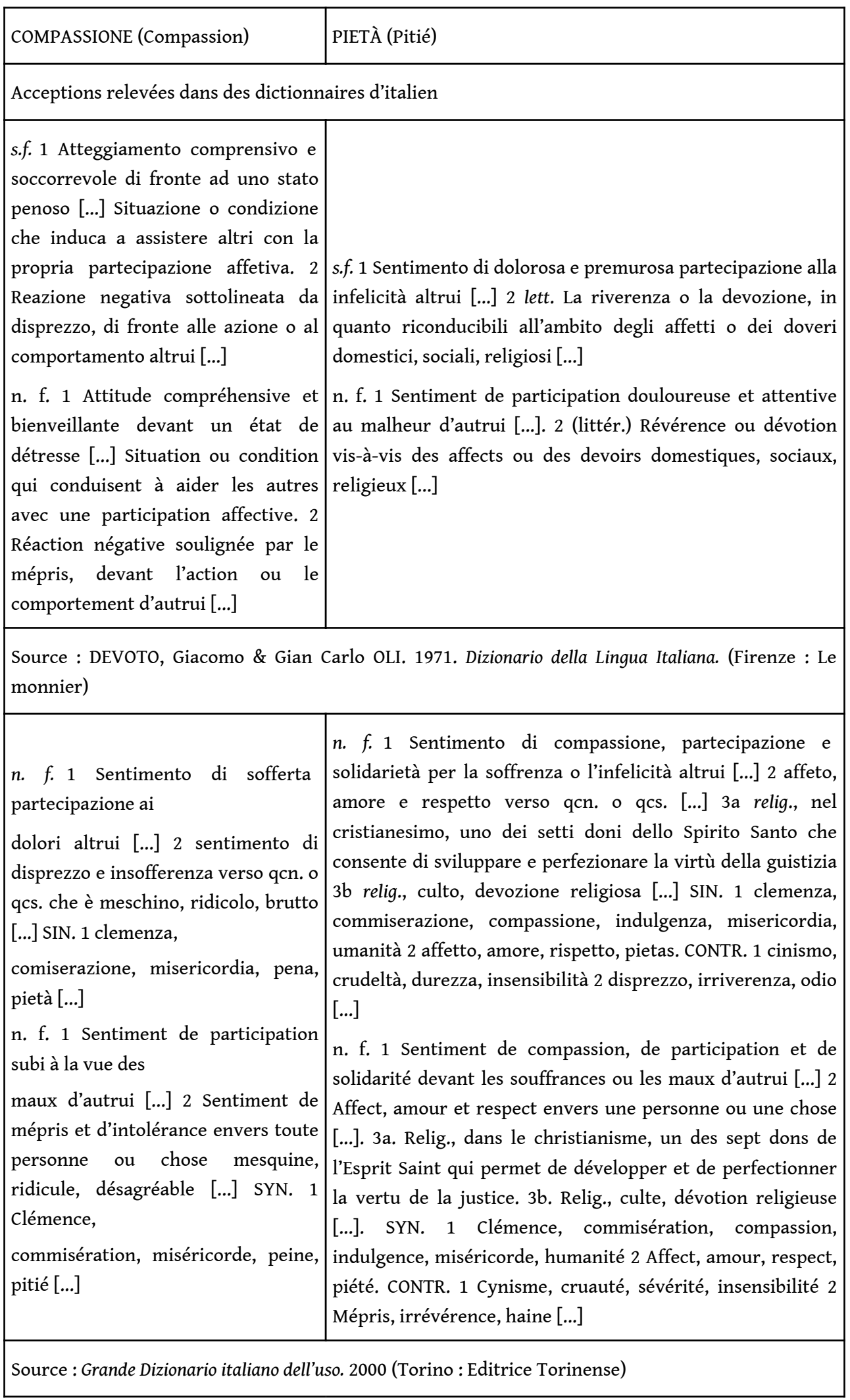

Tableau 3 : Tableau comparatif des définitions relevées dans des dictionnaires d'italien. 
13 Dans les dictionnaires d'espagnol, la compassion et la pitié sont également considérées comme des synonymes. Toutefois, la constance du sème de la religiosité dans la définition de la pitié (voir Tableau 4), en soulignant la connotation de "devoir" (moral) que comprend ce type d'interaction, ne manque pas d'attirer l'attention. Contrairement aux autres dictionnaires examinés, où ce sème n'est pas toujours exprimé, dans le contexte espagnol, sa présence est un fait consolidé. Cette consolidation sous-entend, par comparaison, la nature plus spontanée du sentiment de compassion.

\begin{tabular}{|c|c|}
\hline COMPASIÓN (Compassion) & PIEDAD (Pitié) \\
\hline \multicolumn{2}{|c|}{ Acceptions relevées dans des dictionnaires d'espagnol. } \\
\hline $\begin{array}{l}\text { s/f Lástima, ternura y comprensión que } \\
\text { una persona siente hacia outra que sufre } \\
\text { o tiene problemas [...] SIN. Misericordia, } \\
\text { comiseración, piedad. ANT. } \\
\text { Insensibilidad, crueldad. } \\
\text { n. f. Peine, tendresse et compréhension } \\
\text { ressenties chez une personne pour celui } \\
\text { qui souffre ou connaît des problèmes. } \\
\text { SYN. } 1 \text { Miséricorde, commisération, pitié. } \\
\text { ANT. Insensibilité, cruauté. }\end{array}$ & $\begin{array}{l}\text { s/f } 1 \text { Sentimiento de compasión por alguien que sufre } \\
\text { [...] } 2 \text { Sentimiento de religiosidad y devoción por lo que } \\
\text { es o se considera sagrado o digno de veneración [...] } \\
\text { SIN. } 1 \text { Compasión, clemencia, misericordia. } \\
2 \text { Fervor, devoción. } \\
\text { n. f. Sentiment de compassion éprouvé à l'égard de } \\
\text { celui qui souffre [...] } 2 \text { Sentiment de religiosité et de } \\
\text { dévotion pour ce qui est ou est considéré comme sacré } \\
\text { ou digne de vénération [...] SYN. } 1 \text { Compassion, } \\
\text { clémence, miséricorde. } 2 \text { Ferveur, dévotion. }\end{array}$ \\
\hline \multicolumn{2}{|c|}{$\begin{array}{l}\text { Source : Gran diccionario de uso del español actual. } 1985 \text { (Alcabendas-Madrid : Sociedad General } \\
\text { Española de Librería) }\end{array}$} \\
\hline $\begin{array}{l}f \text {. Sentimiento de commiseración y } \\
\text { lástima que se tiene hacia quienes sufren } \\
\text { penalidades o desgracias. } \\
\text { n. f. Sentiment de commisération et de } \\
\text { peine que nous éprouvons pour ceux qui } \\
\text { souffrent de maux ou de malheurs. }\end{array}$ & $\begin{array}{l}\text { f. Virtud que inspira, por el amor a Dios, tierna } \\
\text { devoción a las cosas santas, y, por el amor al prójimo, } \\
\text { actos de amor y compasión. // } 2 \text { Amor entrañable que } \\
\text { consagramos a los padres y a objetos venerandos. // } 3 \\
\text { Lástima, misericórdia, commiseración [...] } \\
\text { n. f. Vertu qui inspire, par l'amour de Dieu, une tendre } \\
\text { dévotion pour les choses saintes, et, par l'amour du } \\
\text { prochain, des actes d'amour et de compassion. // } 2 \\
\text { Grand amour que nous ressentons pour nos parents et } \\
\text { pour les objets vénérables. // } 3 \text { Peine, miséricorde, } \\
\text { commisération [...] }\end{array}$ \\
\hline
\end{tabular}

Tableau 4 : Tableau comparatif des définitions relevées dans des dictionnaires d'espagnol

14 Cet inventaire des définitions montre deux expériences passionnelles possibles, sousjacentes à l'éveil de l'attendrissement pour les souffrances d'autrui qui suscitent donc divers positionnements et modes d'interaction affective. Il peut en ressortir une identification fusionnelle, de synchronisation pathémique, ou une identification marquée par la position d'altérité, qui maintient une distance et assure une certaine supériorité à celui qui perçoit la souffrance de l'autre, par le fait même de se trouver 
dans une meilleure situation. Dans les termes de Fontanille (2005:241), «dans le premier cas, l'autre est traité comme un semblable dans le mouvement même de participation-identification ; et, dans le second cas, l'évaluation est comparative, et non absolue $[. .$.$] ».$

Cette différence qualitative dans le mode de configuration de l'interaction affective issue de l'observation du mal qui afflige autrui s'éclaircit lorsque nous examinons la racine étymologique des termes en question (voir Tableau 5).

\begin{tabular}{|c|c|}
\hline COMPASSION & PITIÉ \\
\hline \multicolumn{2}{|l|}{ Étymologie } \\
\hline $\begin{array}{l}\text { - } 1155 \text {; lat. chrét. compassio, de compati } \\
\text { « souffrir » compatir } \\
\text { - 1541; bas lat. compati « souffrir avec », } \\
\text { d'apr. Pâtir « souffrir » } \\
1 \text { Vx. S'accorder, être compatible. } 2 \text { Mod. } \\
\text { COMPATIR À : avoir de la compassion pour (une } \\
\text { souffrance). } \rightarrow \text { s'apitoyer, s'attendrir, plaindre. }\end{array}$ & $\begin{array}{l}\text { - } 1080 \text {; lat. pietas, atis } \rightarrow \text { piété } \\
\text { - } 1552 \text {; « pitié » fin Xe ; lat. Pietas } \\
1 \text { Fervent attachement au service de Dieu, aux } \\
\text { devoirs et aux pratiques de la religion. } 2 \text { Littér. } \\
\text { Attachement fait de tendresse et de respect. }\end{array}$ \\
\hline
\end{tabular}

Tableau 5 : Comparaison de la racine étymologique de la compassion et de la pitié

Manifestement, l'identification de deux "types d'attendrissement", qui recouvrent d'une certaine façon, encore aujourd'hui, dans la pratique discursive, le champ sémantique de la compassion et de la pitié, est un vestige étymologique. La compassion, chargée des sèmes du " commun », de la " communion », constitue effectivement, sur la base de ce fondement étymologique, un sentiment plus spontané, d'origine naturelle et instinctive. D'après Ricot $(2013: 13)$ : «Par ce trait, la compassion possède un avantage inscrit dans la structure de son étymologie latine puisqu'elle indique explicitement la co-souffrance : la relation à l'autre, lisible très directement dans le mot lui-même [...] ». La pitié, elle, en mettant en avant le devoir moral et la religiosité/ la vertu - comme en témoignent dans la langue française les traits définitoires de son cousin germain le vocable "piété ", à l'usage plus circonscrit -, dénote l'emprise de la raison dans la configuration affective, la détermination sociale de l'état d'âme, l'obligation du sentir par rapport au mal vécu par autrui; le sentiment de commisération devant la souffrance de l'autre (pitié) dérive d'un devoir-sentir socialement conditionné (piété). Pour un exemple littéraire notoire, rappelons-nous Énée, dans l'Énéide de Virgile, qualifié de "pieux», bien que sa conduite ne manifeste aucun sentiment de compassion.

La différence entre ces deux formes d'attendrissement se manifeste également dans les études réalisées par les philosophes qui ont traité de ce type de sentiment - Smith, Rousseau, Kant, Nietzsche, Schopenhauer, entre autres. L'interaction fondée sur le « sentir avec » est souvent exaltée, alors que la conduite « sentir pour » se trouve, dans la plupart des cas, discréditée. 
i qu'il en soit, une simple interprétation des sèmes ne saurait nous satisfaire. À l'instar de Greimas et Fontanille (1991), nous considérons qu'il faut établir, sur la base des segments définitionnels relevés, les éléments du processus de configuration de ces états d'âme. Ainsi, il convient de saisir les circonstances de la mise en place de l'interaction pathémique, qui font que la compassion se lie davantage à l'identification et à la sensibilisation passionnelle, et suscite une interaction affective plus tonique, un «sentir avec" relevant plutôt de l'ordre du sensible ; alors que la pitié se trouve attachée à une évaluation plus objective et distanciée de la situation de la souffrance d'autrui, à l'altérité et à la moralisation, à l'intelligible, et suscite un "sentir pour ", l'interaction intersubjective étant régie avant tout par l'intelligible.

\section{La structure narrative sous-jacente à la définition}

19 Le point de vue de la sémiotique des passions se caractérise par la complexité, c'est-àdire par la corrélation entre des dispositifs et des dimensions appartenant à différents niveaux de génération du sens, de telle sorte que chaque état passionnel est sous-tendu par une structure narrative formelle. Greimas et Fontanille, dans leur examen de l'avarice, donnent l'explication suivante :

Il s'agit en somme de transformer des rôles pathémiques, dont les'noms-lexèmes' attestent l'existence dans un usage donné, en pathèmes-procès et de mettre en lumière, grâce à l'analyse et la catalyse conjuguées, les organisations modales sousjacentes, ainsi que les opérations qui les prédisposent à participer aux configurations passionnelles. Cette procédure [...] se fonde sur le constat des propriétés de condensation et d'expansion du discours, qui autorisent à déployer, à partir d'un seul lexème, l'ensemble d'une organisation syntaxique (1991 : 111, en italiques dans le texte).

L'examen des conditions de configuration des effets passionnels de la compassion et de la pitié ainsi que la prise en compte de leur distinction nous permettent de privilégier les sèmes susceptibles d'être déduits à partir de la racine étymologique des lexèmes. Nous obtenons alors le tableau suivant :

\begin{tabular}{|l|l|}
\hline COMPASSION & PITIÉ \\
\hline souffrance commune ; communion, participation & accomplissement du devoir ; religiosité, vertu \\
\hline symétrie ; identification & dissymétrie ; altérité \\
\hline « sentir avec » & « sentir pour » \\
\hline
\end{tabular}

Tableau 6 : Description des segments définitionnels des termes en analyse

21 Comme il s'agit, dans les deux cas, d'un sentiment-réponse à la vue de la souffrance d'autrui, un premier constat peut être établi à propos de la similitude du dispositif actantiel. Indépendamment de l'investissement particulier qu'ils reçoivent dans telle ou telle manifestation discursive, il est impératif d'avoir : (1) un mal, (2) un sujet qui en est affecté et (3) un sujet qui observe la scène en question. De surcroît, cette première imposition structurelle nous amène à déduire un autre principe commun entre les deux états d'âme : la relation intersubjective entre les sujets impliqués (d'une part, le sujet 
souffrant, qui peut être conçu comme un objet-valeur de l'affectivité suscitée; de l'autre, le témoin de la souffrance, que nous appellerons sujet de la perception). Cette relation est fondée sur la composante fiduciaire, le "croire ", qui déclenche, dans ce cas, le processus d'interaction pathémique entre les sujets. Pour Fontanille (2005:241) :

Toute cette série comporte deux présupposés indispensables. À savoir : 1. Que l'autre soit dans une situation dysphorique : cela implique évidemment une appréciation de sa situation, au plan thymique, voire axiologique ; [...]. 2. Que l'autre soit reconnu comme un semblable. [...] éprouver de la compassion ou de la pitié, c'est identifier au moins un trait partagé, qui fera de l'autre un semblable [...].

En somme, pour que l'état d'âme compatissant ou s'apitoyant se configure, deux «mécanismes» doivent agir au moment de l'émergence de l'interaction affective (avant tout perceptive) : (1) l'interprétation de la situation dysphorique qui se présente au sujet de la perception et (2) le mouvement d'assimilation, c'est-à-dire l'identification avec le sujet qui souffre, que cette identification soit complète ou partielle. Toujours selon Fontanille (ibid.) : «cette appréciation peut être purement participative (se mettre à la place d'autrui), une identification somatique et sensible ; mais elle peut aussi être évaluative, et c'est alors que la seule manifestation du jugement devient dépréciative (il y entre 'quelque chose du mépris')».

Le sujet compatissant ou s'apitoyant, témoin de la souffrance d'autrui et croyant partager les mêmes valeurs, compatit, en un mouvement de conjonction, car il croitsavoir ce que l'autre souffre, il croit-savoir l'effet causé par le mal qui l'afflige. Du même coup, le croire(-savoir), en établissant, dans la situation de production de ces effets pathémiques, le lien entre le sujet de la perception et le sujet perçu, qui souffre, se présente comme le pivot passionnel de ces interactions affectives, comme un élément d'intersubjectivité. Ce croire(-savoir) est responsable de "l'éveil» de l'expérience pathémique, l'élément qui, en marquant un mouvement d'identification, de reconnaissance, configure la compétence du sujet à sentir.

Comme l'explique Greimas : "la re-connaissance, contrairement à la connaissance, est une opération de comparaison de ce qui lui est'proposé' [...] et de ce qu'il sait/croit déjà » (1983 : 119, en italiques dans le texte). Ainsi, la propriété formelle qui fonde les scènes passionnelles en question s'instaure : le sujet pathémique, qu'il soit compatissant ou s'apitoyant, conçoit, à partir de la manifestation de la souffrance d'autrui et de la comparaison avec soi-même, ce qu'il estime être "l'être " de la souffrance, ce qui fonde l'imaginaire (le "croire-savoir») qui le rend compétent pour sentir. Ce faire interprétatif, en tant qu'opération basée sur la reconnaissance (Greimas 1983), constitue donc l'élément clé de la structuration pathémique de l'effet de compassion et de pitié.

Pour Adam Smith (1999: 24-25) :

nous ne pouvons pas former une idée de la manière dont ils sont affectés qu'en concevant ce que nous devrions nous-mêmes sentir dans la même situation. [...] Ce n'est que par l'imagination que nous pouvons former une conception de ce que sont ses sensations. Et cette faculté ne peut nous y aider d'aucune autre façon qu'en nous représentant ce que pourraient être nos propres sensations si nous étions à sa place. [...] Ses souffrances, quand elles sont ainsi ramenées en nous, quand nous les avons ainsi adoptées et faites nôtres, commencent enfin à nous affecter [...].

L'activité épistémique (le croire), en tant qu'acte cognitif qui sensibilise et pathémise la relation intersubjective, se situe, en ce sens, au cœur de ce type d'interaction affective. En tout état de cause, même s'il existe un principe structurant commun aux deux états 
d'âme - qui saurait peut-être expliquer le fait que les affects en question soient considérés comme des passions synonymes -, l'«être de l'être » ou mieux l'« être du croire " est, pour chacun de ces affects, surdéterminé, comme le démontrent les segments définitionnels décrits précédemment, par différents prédicats modaux : un vouloir(-être) propre au sujet compatissant et un devoir(-être) pour le sujet s'apitoyant.

Nous pouvons inférer que dans la compassion, dont la modalité dominante serait le vouloir, l'interaction entre celui qui souffre et celui qui observe cette souffrance opère plus directement, en marquant ce que nous pouvons nommer une autodestination, c'est-à-dire une identification synchrone propre à une interaction plus sensible, conformément à ce que laissent entendre les sèmes relevés à partir de la racine étymologique. Dans ce cas, le croire-savoir la souffrance de l'autre, l'opération de comparaison, possède une nature tonique et configure une conjonction accomplie - la douleur de l'autre devient la douleur du sujet de la perception lui-même.

En ce qui concerne la pitié, dont la modalité régissante serait le devoir, la relation entre les sujets impliqués serait prédéterminée par la discrétisation, dans l'instauration du sujet s'apitoyant, des fonctions du destinateur (la conduite morale) et du destinataire (le sujet de la perception). De la sorte, le sentir qui pathémise l'interaction de pitié ne provient pas du sujet lui-même, mais d'une imposition (sociale) de commisération, d'une "obligation", d'un "devoir-être» que le destinateur social, collectif, lui prescrirait. Il ne s'agit pas ici d'une interaction affective tonique et sensible, spontanée, comme dans le cas de la compassion, mais plutôt d'une interaction sociale, d'une vertu, de ce fait moins tonique et davantage liée à l'intelligible.

Le croire-savoir des sujets compatissant et s'apitoyant, en tant que déterminant du mode d'interaction affective établi dans une passion ou dans l'autre, pointe donc vers des formes distinctes de configuration de la compétence passionnelle, et par conséquent du lien entre les sujets dans chacun de ces cas. Ainsi, un observateur sensible s'instaure dans le cas de la compassion et un observateur social dans celui de la pitié. À cet égard, Discini (2011) explique que l'observateur sensible est, de fait, autocentré axiologiquement et affectivement, alors que l'observateur social est celui qui se définit par un positionnement socialement prédéterminé. En outre, Greimas et Fontanille (1991: 170) ajoutent que « l'observateur social [...] moralise la manifestation passionnelle pour réaffirmer un état de choses aux dépens d'un état d'âme »; en revanche, l'observateur sensible ne privilégie pas l'état de choses, mais l'état d'âme procédant de la manifestation passionnelle observée - nous y reviendrons.

Le lien affectif établi entre les sujets (un sujet de la perception et un sujet de la souffrance) se caractérise donc par un faire interprétatif (un croire-savoir) de nature qualitative distincte. Le vouloir-être et le devoir-être, en tant que modalités de base de la configuration du sujet compatissant et s'apitoyant, restent attachés aux modes d'existence du croire dans la relation intersubjective et, par conséquent, du sentir en tant que tel. Selon Fontanille et Zilberberg, la croyance, conçue comme une compétence, peut même prendre deux formes :

Cette croyance qui, du point de vue de la portée prédicative, sera endogène, et, du point de vue tensif, ouvrante : c'est l'assomption (le sujet assume sa compétence comme une efficience sentie comme «de l'intérieur», en quelque sorte en toute «autonomie ») ; la seconde est une croyance exogène, et, du point de vue tensif, clôturante : c'est l'adhésion (le sujet adhère à sa compétence comme une efficience sentie comme «de l'extérieur »; on aurait alors affaire à l'« hétéronomie ») (1998 : 190 , en italiques dans le texte). 

pris de compassion ou de pitié, nous approfondirons l'exploration des circonstances de la configuration du croire-savoir-assumé et du croire-savoir-adhéré. Nous nous efforcerons de comprendre les spécificités de la réponse affective plus sensible ou plus intelligible qui émerge de l'interaction entre les sujets impliqués. Pour ce faire, nous examinerons désormais la constitution du champ de (co-)présence dans 
lequel opère l'interaction. Ce champ est considéré comme le domaine spatio-temporel (l'espace-temps subjectif) où s'exerce la perception de la souffrance d'autrui et par conséquent l'interaction (sensible-intelligible) entre le sujet de la perception et l'objetvaleur de l'affectivité suscitée.

L'établissement de cette interaction associe deux présupposés : (1) les modalités d'émergence des valeurs axiologiques qui caractérisent la scène instituée, lesquelles régissent la convocation de la sensibilité et de l'intelligibilité du sujet témoin de la souffrance de l'autre, et, sur cette base, (2) le mode d'institution de la présence d'une telle souffrance.

Ainsi, nous entamerons cette seconde partie de l'analyse avec l'idée de Lopes (2006: 11) sur les conditions de la configuration du champ de (co-)présence :

La profondeur [du champ de (co-)présence] proposée est mesurée par les corrélations entre l'intensité et l'extensité identifiables dans ce champ positionnel. En principe, plus on est éloigné du centre déictique (extensité plus importante), plus l'intensité sensible s'amenuise - sensible signifiant simultanément, dans ce cas, " perceptive » et "thymique "; plus on est proche du centre déictique (extensité moins importante), plus l'impact sensible augmente, donc l'intensité.

Selon cette perspective, toute grandeur perçue qui entre dans le champ de présence du sujet observateur (lorsque le sujet se rend compte de la souffrance d'autrui) se trouve qualifiée à la fois en termes d'intensité (force affective) et d'extensité (étendue de la présence perçue). La première s'évalue à partir de la tonicité de la présence perçue (la souffrance + ou - tonique, + ou - atone) et du tempo (l'appréhension des valeurs perçues + ou - accélérée, + ou - ralentie). La seconde concerne l'ouverture (la diffusion) ou la fermeture (la concentration) de l'espace de perception, du nombre d'éléments en jeu, et concerne donc également la brièveté ou l'allongement du temps de perception de la souffrance, sa temporalité, sa durée. Les effets de l'intensité de cette présence perçue agissent sur la convocation sensible du sujet percevant, sur sa sensibilité, tandis que les effets de l'extensité agissent sur la capacité d'assimilation intelligible de la présence perçue.

Comme nous l'avons constaté - et comme le soulignent déjà les définitions dictionnairiques -, si la compassion est prescrite par une interaction de plus grande sensibilité entre les sujets impliqués, il est établi pour cet état d'âme que les valeurs investies dans la souffrance perçue (quelles qu'elles soient) se manifestent plus vivement, avec une force de convocation sensible plus importante, ce qui renforce la synchronisation entre les personnes impliquées (je perçois la douleur de l'autre comme s'il s'agissait de la mienne). Ceci présuppose une insertion accélérée et tonique des valeurs qui caractérisent la souffrance perçue, un champ de (co-)présence à la profondeur compacte, propre à une contraction de l'espace et de la durée de la perception, à une inhibition conséquente de la durée de traitement intelligible de ce qui est perçu (la douleur de l'autre, je ne l'imagine pas, je la sens).

Dans le cas de la pitié, où les sujets impliqués entretiennent une relation asymétrique, guidée davantage - selon les définitions - par un traitement intelligible de la souffrance d'autrui que par une participation sensible, le champ de présence constitué montrera une plus large profondeur, avec un espace-temps plus important pour la perception. L'émergence des valeurs investies dans la souffrance perçue apparaîtra légèrement moins accélérée, plus distribuée, et présentera donc un impact de tonicité plus faible (il ne s'agit pas seulement des sujets percevant et perçu, mais aussi des circonstances d'un 
tel malheur) ; partant, on peut s'attendre à une plus grande assimilation intelligible de ce qui est perçu. Comme le soulignent Fontanille et Zilberberg (1998: 108) :

Si l'émetteur est tonique, son action apparait comme un «coup » et produit sur le récepteur un «effet»; si l'émetteur est atone, son action apparaît seulement comme "efficience» et le récepteur se contente de la ressentir comme "présence ». Pour l'observateur, l'« effet » et la "présence » manifestent alors respectivement l'« action » et l'« efficience » de l'émetteur.

Ces déterminations de la profondeur du champ de (co-)présence (l'espace subjectif du sujet de la perception), propre à l'émergence de la compassion et/ou de la pitié, confirment et expliquent à nouveau la question de la compétence passionnelle fondée sur le « sentir avec » (émotion intense, tonique, qui génère l'unité, la fusion entre les sujets) chez le sujet compatissant et sur le «sentir pour " (émotion distribuée et donc plus atone, marquant une non-disjonction) chez le sujet s'apitoyant. Les auteurs précités ajoutent également :

La réalisation du sujet $\mathrm{S}$, face à un monde $\mathrm{M}$ perçu comme unique et d'une présence éclatante, le consacre comme contracté, unifié, dans la mesure où il n'y a aucune distance entre la visée et la saisie, entre l'intéroceptivité et la proprioceptivité : l'appropriation du monde $\mathrm{M}$ par le sujet $\mathrm{S}$ est en quelque sorte simultanée avec leur mise en présence. [...] L'actualisation met en présence le sujet $S$ et un monde $M$ perçu comme concentré et massif : elle reconstitue ainsi en partie la tension entre les deux instances S' et S", et rend possible, sinon une synchronisation, du moins une superposition partielle de leurs actes et de leurs rôles respectifs, de sorte que le sujet peut être dit ici mobilisé [...] (1998 : 104, en italiques dans le texte).

Dans cette perspective, le sentir qui rend compatissant le sujet se configure par la présence vive, tonique, de la souffrance perçue, de la douleur de l'autre dans un champ de présence circonscrit et dépouillé de la perception des circonstances autour, motive un lien plus intense, une tension intersubjective plus importante, et, par conséquent, une plus grande mobilisation de la sensibilité du sujet de la perception. Comme l'expliquent Fontanille et Zilberberg (1998: 96) :

"viser", c'est sélectionner dans une étendue ouverte la zone où s'exercera la perception la plus intense; c'est renoncer à l'étendue et au nombre des objets au profit de la saillance perceptive de quelques-uns ou d'un seul [...], l'intensité et l'extensité perceptives évoluent de manière inverse : moins on vise d'objets à la fois, mieux ils sont visés.

L'effet de compassion, rattaché à l'opération de tri, s'ancre donc sur le type perceptif de l'appréhension locale, selon une grandeur caractérisée par une tonicité perceptive élevée et par une exclusivité conséquente dans la perception du sujet compatissant, avec une plus grande homogénéité intersubjective de l'interaction affective déclenchée, propre aux valeurs d'absolu. Face à une tragédie inattendue, par exemple, mon attention se portera tout entière sur le malheur des victimes plutôt que sur les tenants et les aboutissants de la tragédie en tant que telle. Cette appréhension ciblée explique que le croire-savoir se configure comme un croire-savoir-assumé, typique d'une interaction affective effectivement réalisée. Les valeurs investies dans la souffrance de l'autre sont ressenties par le sujet de la perception comme étant de son propre fait ; d'où l'assomption d'un point de vue plus individualisé - «dans la compassion, le fossé entre moi-même et autrui s'évanouit. L'être qui ressent la compassion est en fin de compte identique avec celui dont les peines suscitent de la compassion" (Schopenhauer 1998 : 182). 

totalisation axiologique, relie la tension interactive au fractionnement de l'activité perceptive étendue aux circonstances. Il indique simultanément un degré moindre de tonicité perceptive car celle-ci s'étale sur un espace-temps plus large, plus proche des valeurs d'univers. La tragédie elle-même m'impressionne ; plus je saisis les éléments de son déclenchement, plus je suis en mesure de me figurer la souffrance de ceux qu'elle a frappés. On comprend ainsi l'action de l'intelligible dans la production de l'attendrissement, lequel «procède par la délimitation d'une étendue, et cerne le domaine pour y circonscrire l'objet» (Fontanille et Zilberberg, 1998 : 95). Les valeurs investies dans l'attendrissement de la pitié, mélangées à celles qui caractérisent les circonstances d'où procède la souffrance de l'autre, sont ainsi appréhendées comme extérieures. Elles sont certes reconnues par le sujet de la perception, mais sont considérées comme relevant de la seule situation du sujet qui souffre; ce qui explique la symétrie moindre des places respectives de ces deux sujets et l'adhésion à un point de vue moins individuel. Bien qu'une certaine intensité puisse s'y configurer, l'extensité propre à l'effet de pitié multiplie les éléments perçus et déstabilise l'homogénéité ainsi que la symétrie entre les sujets impliqués.

Dans ce cadre, la distinction entre les sujets compatissant et s'apitoyant ne réside pas nécessairement dans le degré d'intensité du sentir, mais surtout dans la variation de la tonicité de la présence de la souffrance perçue, dans la façon dont on y accède : directement ou indirectement. Cette proposition de distinction est conforme à ce que soutient Christine Tappolet (2000 : 177-178), qui, sur la base de la distinction « empathy » et « sympathy » de Sober et Wilson (1998), affirme : « la différence entre la pitié et la compassion est due à ce qu'il est possible de prendre en pitié une personne qui demeure inconsciente de son malheur, alors que la compassion requiert que la personne avec qui l'on compatit soit non seulement consciente de son malheur, mais en soit affectée ».

L'interprétation que nous avançons diffère quelque peu de celle proposée par Fontanille $(2003,2005)$, lorsqu'il souligne, à partir des considérations de Heidegger sur la "préoccupation mutuelle ", que la différence entre la compassion et la pitié repose sur des déterminations aspecto-temporelles. Pour le sémioticien cité, «la gamme passionnelle se diversifie et se définit donc, selon que l'on vise dans l'existence d'autrui son devenir, sa situation ou sa destinée » (Fontanille 2005 : 246, en italiques dans le texte). Il explique (244) :

[...] on peut bien éprouver de la compassion pour tout ce qui attend l'autre dans cette " vallée de larmes » qu'est notre monde, sans pour autant éprouver pour lui de la pitié ; si même on éprouvait en ce cas de la pitié, ce serait au prix d'un considérable effort d'actualisation imaginaire de l'à-venir, ou même au nom de quelque fatalisme qui considérerait le non-accompli (dans le monde concret) comme déjà advenu (dans le monde écrit du destin). De la compassion à la pitié, il se produit donc une réduction des situations possibles à une situation-occurrence réalisée. La compassion porte sur le "pouvoir être ", à la différence de la pitié qui ne peut porter que sur un état actuel.

Chez Fontanille (2005), la distinction compassion/pitié tient donc au mode d'existence de la souffrance d'autrui : possibilité envisagée ou fait accompli que je constate. De notre point de vue, il faut dans un cas comme dans l'autre que le dommage provoquant la souffrance soit accompli, l'écart s'expliquant en fonction de ce qui mobilise le plus fortement mon attention, à savoir, le chagrin de l'autre que je saisis ou bien, au 
contraire, la situation qui provoque la souffrance et qui me permet d'inférer la douleur ressentie. Mais, que l'on veuille raisonner à partir du régime de la temporalité des situations en question, ou à partir du mode de présence de l'objet-valeur de l'attendrissement dans le champ de (co-)présence créé entre les sujets, l'évaluation ne peut se faire que par une analyse où les interactions pathémiques sont examinées in praesentia, c'est-à-dire lorsqu'elles sont manifestées.

\section{Conclusion}

À partir des éléments sémantiques relevés dans les définitions de la compassion et de la pitié, nous avons cherché à saisir, au moins de deux façons, la configuration sousjacente à ces états d'âme, en ce qui concerne (1) l'identité modale de l'attendrissement des sujets compatissant et s'apitoyant, et (2) les conditions d'émergence des affects en question, avec les modulations de l'intelligible et du sensible, qui gèrent l'acte perceptif et instaurent l'interaction affective.

Dans la perspective des modalisations existentielles, les modalités régissent l'identité des sujets compatissant et s'apitoyant, respectivement le vouloir-être et le devoir-être, et révèlent une différence significative dans la structuration du pivot passionnel (le croire-savoir la douleur d'autrui) des deux états d'âme, en termes non seulement structuraux, mais aussi sémantiques : l'interaction compatissante montre un point de vue plus individualisé (valeurs d'absolu, affectivité concentrée), procédant de l'intime humain, propre à une sanction passionnelle de caractère endogène, un croire-savoirassumé, fruit de l'autodestination dans la figure du compatissant ; le sujet s'apitoyant, lui, manifeste un point de vue plus généralisant (valeurs d'univers, affectivité diffuse), lié aux devoirs sociaux, moraux et religieux, avec une sanction d'ordre exogène, un croire-savoir-adhéré.

Ces différences qualitatives du pivot passionnel, le croire-savoir, sont effectivement apparues comme le produit de la façon dont la souffrance est perçue. Nous constatons ainsi que la compassion s'établit lorsque la perception du sujet porte exclusivement sur celui qui souffre, sur la manifestation explicite de sa souffrance, voire sur le désespoir vécu et affiché, lequel concentrerait les valeurs (sémantiques et axiologiques) en jeu et, par conséquent, l'intensité qui convoque et affecte le sujet de la perception, en justifiant la plus grande incitation de sa sensibilité, la configuration du croire-savoirassumé : plus l'emphase sur la souffrance est importante, moindre est le nombre d'éléments perçus pris en considération. En revanche, la pitié procèderait d'une appréhension qui privilégie les circonstances (le «mal causal»), la situation qui faitêtre la souffrance, les valeurs étant surtout typifiées dans l'extension du domaine spatio-temporel perçu. Dans ce cas, la douleur de l'autre atteint le sujet de la perception par catalyse, telle une inférence découlant de l'espace plus ouvert parcouru par la perception. L'ouverture du champ perçu, qui inclut alors les circonstances, compromet l'appropriation plus directe de la souffrance, l'assimilation synchrone et le partage entre les sujets, en distendant également le sentir correspondant à la tension intersubjective. Comme l'attention se porte sur les circonstances (sur les causes), la souffrance s'en trouve déduite ; partant, la sensibilisation est de nature plus intelligible, relevant d'un croire-savoir-adhéré, avec une force de convocation sensible moins importante. 
commotion se manifeste donc tout autant dans la compassion que dans la pitié Cependant, son degré d'action dans la configuration de la sanction intersubjective formulée par le sujet de la perception varie. La façon de percevoir l'objet de l'affectivité ou, plus précisément, le mode d'appréhension des valeurs sémantico-axiologiques qui y sont inscrites apparaît effectivement comme le responsable des conditions de production des caractéristiques typiques de telle ou telle façon d'interagir, de se mettre en rapport (affectivement) avec ce que l'autre représente.

\section{BIBLIOGRAPHY}

Bertrand, Denis. 2000. Précis de sémiotique littéraire (Paris : Nathan)

Discini, Norma. 2011. "Um algoritmo da percepção: o sujeito do afeto". In : Marchezan, Renata Coelho, Cortina, Arnaldo \& Baquião, Rubens César (éds.) A abordagem dos afetos na semiótica. (São Carlos, SP : Pedro \& João editores). 149-172.

Fontanille, Jacques. 2005. « Pitié ». Rallo-Ditche, Elisabeth, Jacques Fontanille, Patrizia Lombardo. Dictionnaire des passions littéraires (Paris : Belin), 240-265

Fontanille, Jacques. 1999. Sémiotique du discours (Limoges : PULIM)

Fontanille, Jacques \& Claude Zilberberg. 1998. Tension et signification (Liège : Mardaga)

Greimas, Algirdas Julien. 1983. Du sens II (Paris : Seuil)

Greimas, Algirdas Julien \& Jacques Fontanille. 1991. Sémiotique des passions. Des états de choses aux états d'âme (Paris : Seuil)

Lopes, Ivã Carlos. 2006. « A noção de 'Profundidad' na semiótica »,CASA. Cadernos de Semiótica Aplicada, v. 4, nº 2, 2006. http://seer.fclar.unesp.br/casa/article/view/564/485

Ricot, Jacques. 2013. Du bon usage de la compassion (Paris: PUF)

Rousseau, Jean-Jacques. 1969. Émile, ou de l'éducation. Oeuvres complètes, vol. IV (Paris : Gallimard, Bibliothèque de la Pléiade)

Schopenhauer, Arthur. 1998 [1840]. Les deux problèmes fondamentaux de l'éthique. Trad. C. Jaedicke (Paris : Editions Alive)

Smith, Adam. Théorie des sentiments moraux. Trad. M. Biziu, C. Gautier, J.-F. Pradeau (Paris : PUF)

Sober, Elliot \& David Sloan Wilson. 1998. Unto Others: the Evolution and Psychology of Unselfish Behavior (Cambrigde, Mass.: Harvard UP)

Tappolet, Christine. 2000. « Compassion et altruisme », Studia philosophica 59, 175-193 


\section{NOTES}

1. Il s'agit de l'une des branches de la sémiotique discursive développée par Algirdas Julien Greimas, consolidée par la publication de Sémiotique des passions. Des états de choses aux états d'âme (1991), ouvrage écrit en collaboration avec Jacques Fontanille.

2. Les traductions des définitions relevées dans les dictionnaires et présentées avec leurs textes originaux sont de notre fait.

\section{ABSTRACTS}

Through a lexical-based analysis, this article examines the points of similarity and, above all, of divergence, in the production of the feelings of compassion or pity. It starts with an investigation based on the dictionary entries of different Romance languages in order to infer the semes that constitute each passionate nucleus, as well as the elements of the process of discourse configuration. By unfolding the narrative structure underlying every lexeme-affection, we illuminate what determines two modes of different affective interactions through the same predicative scene : a more sensitive one, which pertains to a "feeling with", and a more intelligible one, typical of a "feeling for"nh v.

Le propos de notre travail est d'examiner, sur la base d'une analyse lexicale, les points de similitude et surtout de divergence dans l'émergence du sentiment de compassion et de pitié. À cet effet, nous relèverons tout d'abord les définitions dictionnairiques avancées par plusieurs langues romanes, afin d'y saisir les sèmes constituants pour chacun de ces noyaux passionnels ainsi que les éléments de leur processus de configuration discursive. Il s'agit de comprendre, en déployant la structure narrative sous-jacente à chacun des lexèmes-affects, ce qui, à partir d'une même scène prédicative, détermine deux modes distincts d'interaction affective : l'un plus sensible, propre à un « sentir avec », l'autre plus intelligible, typique d'un « sentir pour ».

\section{INDEX}

Mots-clés: attendrissement, compassion, interaction affective, pitié

Keywords: affective interaction, compassion, emotion, pity

\section{AUTHOR}

\section{ELIANE SOARES DE LIMA}

Université de São Paulo, Brésil 